\title{
Fidelity as a marker of topological phase transitions in 2D Dirac materials
}

\author{
J. C. Bolívar \\ Departamento de Fúsica Atómica, Molecular y Nuclear and \\ Instituto Carlos I de Física Teórica y Computacional, \\ Universidad de Granada, Fuentenueva s/n 18071 Granada, Spain
}

N. A. Cordero

Departamento de Física and International Research Centre in Critical Raw Materials for Advanced Industrial Technologies (ICCRAM), Universidad de Burgos, Burgos, Spain and Instituto Carlos I de Física Teórica y Computacional, Universidad de Granada, Granada, Spain

\author{
Á. Nagy \\ Departament of Theoretical Physics, \\ University of Debrecen, Debrecen, Hungary \\ E. Romera
}

Instituto Carlos I de Física Teórica y Computacional and Departamento de Física Atómica, Molecular y Nuclear, Universidad de Granada, Fuentenueva s/n 18071 Granada, Spain 


\begin{abstract}
Silicene, as other 2D buckled structures, is a gapped Dirac material with intrinsic spin-orbit coupling whose band structure can be controlled by applying a perpendicular electric field. It presents a topological phase transition from a topological insulator to a band insulator at the charge neutrality point. We present in this paper a characterization of this phase transition by using fidelity of Loschmidt echoes when a magnetic and two slightly different electric fields are applied by considering the time evolution of two kinds of wave packets, one with a single Gaussian profile and the other with a double Gaussian profile creating a cat state. We also show that Zitterbewegung, classical and revival Loschmidt periods diverge close to but not exactly at the charge neutrality point and explain this behaviour.
\end{abstract}




\section{INTRODUCTION}

Silicene is a two dimensional crystal of silicon, belonging to a group of 2D gapped Dirac materials analogous to graphene but with a relevant intrinsic spin-orbit coupling (as compared to graphene) and a buckled structure. It has been studied theoretically [1, 2] and experimentally [3-7]. Other gapped Dirac materials are germanene, stannene and $\mathrm{Pb}[8]$. In the case of silicene, the low energy electronic properties can be described by a Dirac Hamiltonian with a Fermi velocity $v_{\mathrm{F}}=5 \times 10^{5} \mathrm{~m} \mathrm{~s}^{-1}$ and a value of the spin-orbit coupling $\Delta_{\text {so }}=4.2 \mathrm{meV}$. It has a buckling length $l=0.22 \AA$ which allows to control the band structure by applying an electric field, $\mathcal{E}_{z}=\Delta_{z} / l$, perpendicular to the silicene monolayer generating a tunable band gap $\left|\Delta_{s, \xi}\right|=\left|\left(\Delta_{z}-s \xi \Delta_{\mathrm{so}}\right) / 2\right|(s$ and $\xi$ denote spin and valley, respectively). Silicene has a topological phase transition (TPT) [9] from a topological insulator (TI, $\left|\Delta_{z}\right|<\Delta_{\text {so }}$ ) to a band insulator (BI, $\left|\Delta_{z}\right|>\Delta_{\text {so }}$ ), at a charge neutrality point $(\mathrm{CNP}) \Delta_{z}^{(0)}=s \xi \Delta_{\mathrm{so}}$, with a gap cancellation between the perpendicular electric field and the spin-orbit coupling, thus exhibiting a semimetal behavior.

Topological phases are characterized by topological charges like Chern numbers. Theoretical information measures and uncertainty relations have been used to characterize TPTs [10-13]. Recently, we have studied the time evolution of electron wave packets in silicene under perpendicular magnetic and electric fields to characterize topological-band insulator transitions [14].

In this paper we want to use fidelity to characterize TPTs. Fidelity (also known as Loschmidt Echo), was introduced by Peres [15] and it has been used to understand the dynamics in few [16-18] and many-body [19-21] quantum systems. It has been relevant to quantify a very important problem in quantum physics, that is, the problem of the coherence and stability of the dynamics of many bodies subject to external perturbations [22]. Additionally, fidelity has been used to quantify corruption in the context of quantum information [23]. Recently, it has been used to study quantum phase transitions in a XXZ spin chain model in a random magnetic field [24] and in a spin-interacting XY model [25]. Additonally, we want to point out the seminal article [26], and other interesting works like $[27,28]$ or the revision about Loschmidt echoes and quatum phase transtions in [29].

The paper is organized as follows. In Section 2, we introduce the low energy Hamiltonian describing the electronic properties of silicene and similar 2D materials in perpendicular elec- 


\begin{tabular}{|c|c|c|c|}
\hline & $\Delta_{\mathrm{So}}(\mathrm{meV})$ & $l(\AA)$ & $v_{\mathrm{F}}\left(10^{5} \mathrm{~m} / \mathrm{s}\right)$ \\
\hline $\mathrm{Si}$ & 4.2 & 0.22 & 4.2 \\
$\mathrm{Ge}$ & 11.8 & 0.34 & 8.8 \\
$\mathrm{Sn}$ & 36.0 & 0.42 & 9.7 \\
$\mathrm{~Pb}$ & 207.3 & 0.44 & - \\
\hline
\end{tabular}

TABLE I: Approximate values of model parameters $\Delta_{\text {so }}$ (spin-orbit coupling), $l$ (interlattice distance) and $v_{\mathrm{F}}$ (Fermi velocity) for two-dimensional $\mathrm{Si}, \mathrm{Ge}, \mathrm{Sn}$ and $\mathrm{Pb}$ sheets. These data have been obtained from first-principles computations in [8] $\left(\Delta_{\text {so }}\right.$ and $\left.l\right)$ and $[30,31]\left(v_{\mathrm{F}}\right)$.

tric and magnetic fields. Section 3 contains a study of the relationship between topological phase transitions and fidelity. Our results for Loschmidt periodicities are given in Section 4 while a more detailed study of the time evolution of the echos is presented in Section 5 . Finally, Section 6 contains our conclusions.

\section{LOW ENERGY HAMILTONIAN}

Let us consider a monolayer silicene film in external magnetic $B$ and electric $\mathcal{E}_{z}$ fields applied perpendicularly to the silicene plane. The low energy effective Hamiltonian in the vicinity of the Dirac point is given by [9]

$$
H_{s}^{\xi}=v_{\mathrm{F}}\left(\sigma_{x} p_{x}-\xi \sigma_{y} p_{y}\right)-\frac{1}{2} \xi s \Delta_{\mathrm{so}} \sigma_{z}+\frac{1}{2} \Delta_{z} \sigma_{z}
$$

where $\xi$ corresponds to the inequivalent corners $K(\xi=1)$ and $K^{\prime}(\xi=-1)$ of the first Brillouin zone, respectively, $\sigma_{j}$ are the usual Pauli matrices, $v_{\mathrm{F}}$ is the Fermi velocity of the Dirac fermions (see Table I for theoretical estimations for Si as well as for other materials: $\mathrm{Ge}, \mathrm{Sn}$ and $\mathrm{Pb}$ ), spin up and down values are represented by $s= \pm 1$, respectively, and $\Delta_{\text {so }}$ is the band gap induced by intrinsic spin-orbit interaction, which provides a mass to the Dirac fermions. We are considering the application of a constant electric field $\mathcal{E}_{z}$ which creates a potential difference $\Delta_{z}=l \mathcal{E}_{z}$ between sub-lattices. The value $l$ appears in table I for different materials. The values of the spin-orbit energy gap induced by the intrinsic spinorbit coupling has been theoretically estimated [8, 32-34] for different 2D Dirac materials that we show in Table I. 
The eigenvalue problem can be easily solved. Using the Landau gauge, $\vec{A}=(0, B x, 0)$, the corresponding eigenvalues and eigenvectors for the $K$ and $K^{\prime}$ points are given by $[9-13,35]$

$$
E_{n, \Delta_{z}}^{s, \xi}=\left\{\begin{array}{l}
\operatorname{sgn}(n) \sqrt{|n| \hbar^{2} \omega^{2}+\Delta_{s, \xi}^{2}}, \quad n \neq 0, \\
-\xi \Delta_{s \xi}, \quad n=0
\end{array}\right.
$$

and

$$
|n\rangle_{s, \xi}=\left(\begin{array}{c}
-i A_{n, \Delta_{z}}^{s, \xi}|| n\left|-\xi_{+}\right\rangle \\
B_{n, \Delta_{z}}^{s, \xi}|| n\left|-\xi_{-}\right\rangle
\end{array}\right)
$$

where we denote by $\xi_{ \pm}=(1 \pm \xi) / 2$, the Landau level index $n=0, \pm 1, \pm 2, \ldots$, the cyclotron frequency $\omega=v_{\mathrm{F}} \sqrt{2 e B / \hbar}$, the lowest band gap $\Delta_{s, \xi} \equiv\left(\Delta_{z}-s \xi \Delta_{\mathrm{so}}\right) / 2$ and the constants $A_{n, \Delta_{z}}^{s, \xi}$ and $B_{n, \Delta_{z}}^{s, \xi}$ are given by [35]

$$
\begin{aligned}
& A_{n, \Delta_{z}}^{s, \xi}= \begin{cases}\operatorname{sgn}(n) \sqrt{\frac{\left|E_{n, \Delta_{z}}^{s, \xi}\right|+\operatorname{sgn}(n) \Delta_{s, \xi}}{2\left|E_{n, \Delta_{z}}^{s, \xi}\right|}}, \quad n \neq 0, \\
\xi_{-}, \quad n=0,\end{cases} \\
& B_{n, \Delta_{z}}^{s, \xi}= \begin{cases}\sqrt{\frac{\left|E_{n, \Delta_{z}}^{s, \xi}\right|-\operatorname{sgn}(n) \Delta_{s, \xi}}{2\left|E_{n, \Delta_{z}}^{s \xi}\right|},} \quad n \neq 0, \\
\xi_{+}, \quad n=0,\end{cases}
\end{aligned}
$$

The vector $|m\rangle$ with $m=0,1,2, \ldots$ denotes an orthonormal Fock state of the harmonic oscillator, and we will have $m=|n| \pm \xi_{ \pm}$. Note that the case $m=-1$ always cancels due to the value of the coefficients $A_{n, \Delta_{z}}^{s, \xi}$ or $B_{n, \Delta_{z}}^{s, \xi}$ defined in (4).

\section{FIDELITY AND TOPOLOGICAL PHASE TRANSITIONS}

We are going to generalize the definition of fidelity given by [36] for two slightly different initial states in the following way. If we have a Hamiltonian $H$ and consider a small perturbation that gives a perturbed Hamiltonian $H^{\prime}$, with time evolution operators $U(t)$ and $U^{\prime}(t)$, respectively and study the time evolution of some arbitrary but similar initial states $\left|\Psi_{0}\right\rangle$ and $\left|\Psi_{0}^{\prime}\right\rangle$ with both Hamiltonians, we get

$$
\begin{array}{r}
|\Psi(t)\rangle=U(t)\left|\Psi_{0}\right\rangle \\
\left|\Psi^{\prime}(t)\right\rangle=U^{\prime}(t)\left|\Psi_{0}^{\prime}\right\rangle
\end{array}
$$

The time-dependent fidelity amplitude with respect to the unperturbed evolution, is defined as the overlap of the perturbed and unperturbed time-evolving states:

$$
m(t)=\left\langle\Psi(t) \mid \Psi^{\prime}(t)\right\rangle=\left\langle\Psi_{0}\left|U(-t) U^{\prime}(t)\right| \Psi_{0}^{\prime}\right\rangle .
$$


Time-dependent fidelity is the square of its modulus

$$
M(t)=|m(t)|^{2} .
$$

We will have $M(t)=1$ if $H^{\prime}=H$ and $\left|\Psi_{0}^{\prime}\right\rangle=\left|\Psi_{0}\right\rangle$. It is clear that if the Hamiltonians and the initial states are very similar, i.e. the perturbation is small, we will have a fidelity close to one. Otherwise the fidelity value will be smaller than 1 .

We can also define time-independent fidelity as

$$
F=M(t=0)
$$

If, instead of using states, we want to use densities, we can consider the DFT fidelity amplitude defined by [37]:

$$
f(\rho, \sigma)=\int \rho^{1 / 2}(\mathbf{r}) \sigma^{1 / 2}(\mathbf{r}) d \mathbf{r}
$$

where $\rho(\mathbf{r})$ and $\sigma(\mathbf{r})$ are two densities. We will have $f(\rho, \sigma)=1$ if $\rho(\mathbf{r})=\sigma(\mathbf{r})$ everywhere. The more the densities differ, the smaller the value of the fidelity amplitude.

In order to compute fidelity, we have to write first the Hamiltonian eigenstates given in Eq. (3) in position and momentum representations. We know that Fock (number) states $|n\rangle$ can be written in position representation as

$$
\langle x \mid n\rangle=\frac{\omega^{1 / 4}}{\sqrt{2^{n} n ! \sqrt{\pi}}} e^{-\omega x^{2} / 2} H_{n}(\sqrt{\omega} x)
$$

where $H_{n}(x)$ are the Hermite polynomials of degree $n$. We will introduce the number-state density in position space as $\rho_{n}(x)=|\langle x \mid n\rangle|^{2}$, which are normalized according to $\int \rho_{n}(x) d x=$ 1. Now, taking into account Eq. (3), the position density for the Hamiltonian eigenvectors is given by

$$
\rho_{n, \Delta_{z}}^{s, \xi}(x)=\left(A_{n, \Delta_{z}}^{s, \xi}\right)^{2}\left|\left\langle x|| n \mid-\xi_{+}\right\rangle\right|^{2}+\left(B_{n, \Delta_{z}}^{s, \xi}\right)^{2}\left|\left\langle x|| n \mid-\xi_{-}\right\rangle\right|^{2} .
$$

We will study $f\left(\rho_{n, \Delta_{z}}^{s, \xi}, \rho_{n, \Delta_{z}^{\prime}}^{s, \xi}\right)$ that is, fidelity amplitude for the densities corresponding to electric field values $\Delta_{z}$ and $\Delta_{z}^{\prime}=\Delta_{z}+\delta_{z}$, where $\delta_{z} \ll \Delta_{z}$.

As already stated, there is a prediction (see e.g. [32-34, 38]) that when the gap $\left|\Delta_{s, \xi}\right|$ vanishes at the $\operatorname{CNP} \Delta_{z}^{(0)}$, silicene undergoes a phase transition from a topological insulator (TI, $\left|\Delta_{z}\right|<\Delta_{\text {so }}$ ) to a band insulator (BI, $\left|\Delta_{z}\right|>\Delta_{\text {so }}$ ). This topological phase transition entails an energy band inversion. Indeed, in Figure 1 we show the fidelity amplitude as a function of the external electric potential $\Delta_{z}$ for $B=0.01 \mathrm{~T}$. One can see that there is a 
band inversion for the $n=0$ Landau level (either for spin up and down) at both valleys. The energies $E_{0}^{1, \xi}$ and $E_{0}^{-1, \xi}$ have the same sign in the BI phase and different sign in the TI phase, thus distinguishing both regimes.

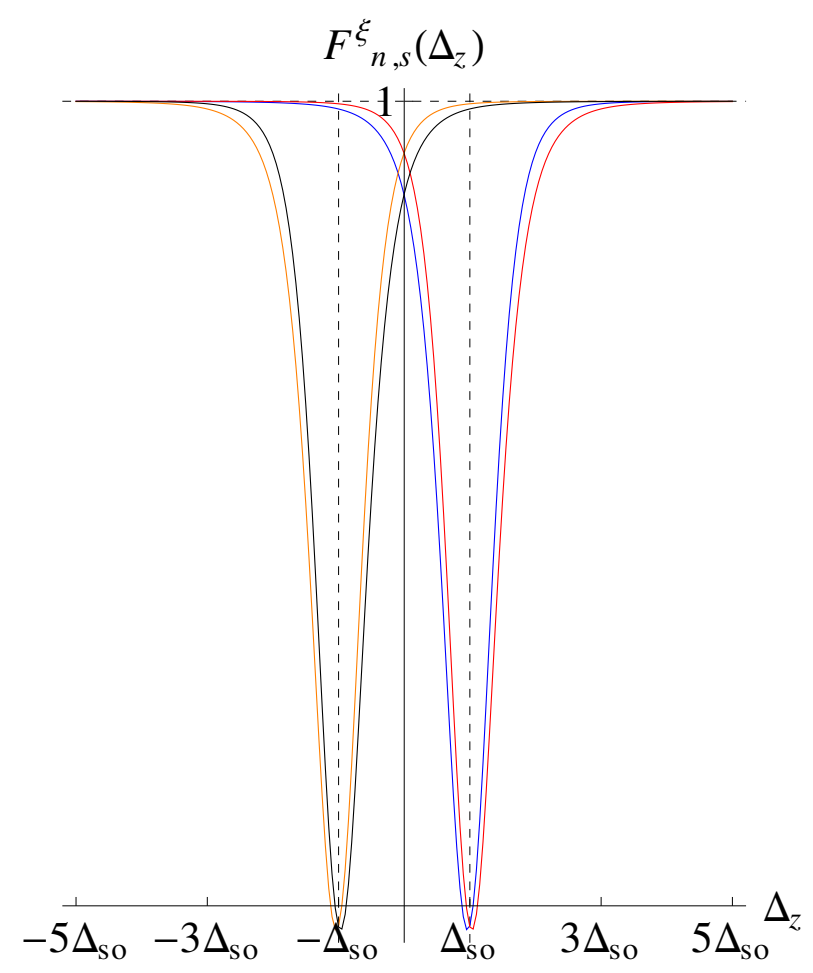

FIG. 1: $F_{n, s}\left(\Delta_{z}\right) \equiv f\left(\rho_{n, \Delta_{z}}^{s \xi}, \rho_{n, \Delta_{z}+\delta \Delta_{z}}^{s \xi}\right)$ when $B=0.01 \mathrm{~T}$ and $\xi=1$ for $n=1, s=1$ (blue); $n=-1, s=1$ (red); $n=1, s=-1$ (orange); and $n=-1, s=-1$ (black).

We are going to consider two types of Gaussian wave packets in this work. The first type is a single Gaussian packet centered on the $n_{0}$-th level above the Fermi level:

$$
\left|\Psi\left(\Delta_{z}, t\right)\right\rangle=|\psi(t)\rangle_{s, \xi}=\sum_{n=0}^{\infty} c_{n} e^{-i E_{n, \Delta z}^{s, \xi} t / \hbar}|n\rangle_{s, \xi}
$$

with

$$
c_{n}=\frac{1}{\sigma \sqrt{\pi}} e^{-\left(n-n_{0}\right)^{2} /\left(2 \sigma^{2}\right)},
$$

where $n_{0}$ defines the center of the wave packet and $\sigma$ its width.

The second type of wave packet is a cat state that consists of two Gaussians symetrically distributed around the $n_{0}$-th levels above and below the Fermi level:

$$
\left|\Psi\left(\Delta_{z}, t\right)\right\rangle=|\psi(t)\rangle_{s, \xi}=\sum_{n=0}^{\infty} c_{n} e^{-i E_{n, \Delta z}^{s, \xi} t / \hbar}|n\rangle_{s, \xi}+
$$



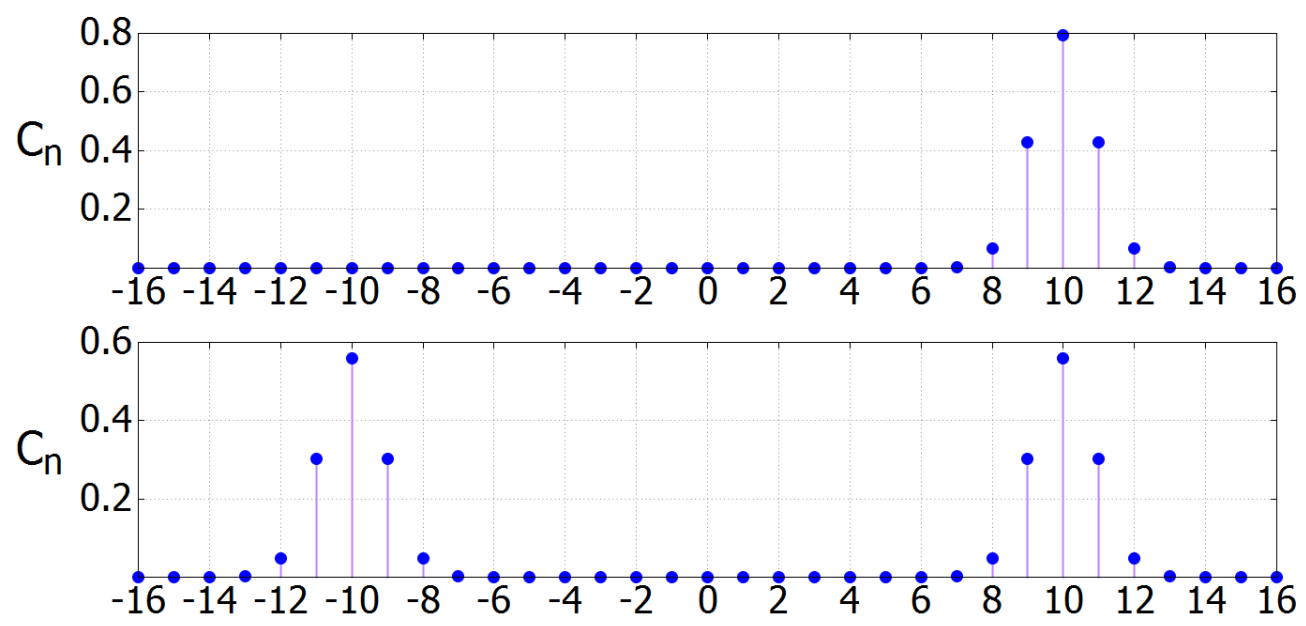

FIG. 2: Coefficients used for the two types of wave packets used. The upper panel shows the packet centered around $n_{0}=10$ (we will call it Type I) while the lower panel depicts the packet centered around $n_{0}=10$ and $n_{0}^{\prime}=-n_{0}=-10$ (we will call it Type II). In both cases $\sigma=0.9$.

$$
+\sum_{n=-\infty}^{0} c_{n}^{\prime} e^{-i E_{n, \Delta z}^{s, \xi} t / \hbar}|n\rangle_{s, \xi}
$$

with

$$
c_{n}=\frac{1}{\sigma \sqrt{2 \pi}} e^{-\left(n-n_{0}\right)^{2} /\left(2 \sigma^{2}\right)} \quad \text { and } \quad c_{n}^{\prime}=\frac{1}{\sigma \sqrt{2 \pi}} e^{-\left(n+n_{0}\right)^{2} /\left(2 \sigma^{2}\right)} .
$$

Examples of coefficients for both types of wave packets -that we will call Type I and Type II, respectively- can be seen in Fig. 2.

Time-dependent fidelity can be written for a Type I packet as

$$
M_{\mathrm{L}}^{s, \xi}\left(\Delta_{z}, \delta_{z}, t\right)=\left|m_{s, \xi}\left(\Delta_{z}, \delta_{z}, t\right)\right|^{2}
$$

where

$$
\begin{gathered}
m_{s, \xi}\left(\Delta_{z}, \delta_{z}, t\right)\left\langle\Psi\left(\Delta_{z}^{\prime}, t\right) \mid \Psi\left(\Delta_{z}, t\right)\right\rangle= \\
={ }_{\Delta_{z}^{\prime}}\left\langle\Psi_{0}\left|e^{i H\left(\Delta_{z}^{\prime}\right) t / \hbar} e^{-i H\left(\Delta_{z}\right) t / \hbar}\right| \Psi_{0}\right\rangle_{\Delta_{z}}= \\
=\sum_{n, m=0}^{\infty} c_{m} c_{n} e^{i\left(E_{m, \Delta_{z}^{s, \xi}}^{\left.s,-E_{n, \Delta_{z}}^{s, \xi}\right) t / \hbar}{ }_{s, \xi, \Delta_{z}^{\prime}}\langle m \mid n\rangle_{s, \xi, \Delta_{z}}\right.} .
\end{gathered}
$$

Since we are only going to consider very small $\delta_{z}$ values, we can assume the variations in the energy levels due to the small change in the electric field are smaller than the difference between two consecutive levels and the orthogonality relations among states will approximately 
hold, i.e. ${ }_{s, \xi, \Delta_{z}^{\prime}}\langle m \mid n\rangle_{s, \xi, \Delta_{z}} \approx{ }_{s, \xi, \Delta_{z}}\langle m \mid n\rangle_{s, \xi, \Delta_{z}}$ with

$$
\begin{gathered}
{ }_{s, \xi, \Delta_{z}}\langle m \mid n\rangle_{s, \xi, \Delta_{z}}=A_{m, \Delta_{z}^{\prime}}^{s, \xi} A_{n, \Delta_{z}}^{s, \xi} \delta_{|m|-\xi_{+},|n|-\xi_{+}}+B_{m, \Delta_{z}^{\prime}}^{s, \xi} B_{n, \Delta_{z}}^{s, \xi} \delta_{|m|-\xi_{-},|n|-\xi_{-}}= \\
=A_{m, \Delta_{z}^{s}}^{s, \xi} A_{n, \Delta_{z}}^{s, \xi} \delta_{|m|,|n|}+B_{m, \Delta_{z}^{\prime}}^{s, \xi} B_{n, \Delta_{z}}^{s, \xi} \delta_{|m|,|n|} .
\end{gathered}
$$

Taking into account the Kronecker deltas in Eq. (18) the fidelity time evolution given in Eq. (17) is governed by the energy differences $E_{n, \Delta_{z}^{\prime}}^{s, \xi}-E_{m, \Delta_{z}}^{s, \xi}$ for $m=n$. We are going to assume levels are so close to each other we can use the continuous approximation and perform a limited Taylor expansion for the energy around the $n_{0}$ level,

$$
\begin{array}{rc}
E_{n, \Delta_{z}^{\prime}}^{s, \xi}-E_{n, \Delta_{z}}^{s, \xi} & \approx\left(E_{n_{0}, \Delta_{z}^{\prime}}^{s, \xi}-E_{n_{0}, \Delta_{z}}^{s, \xi}\right)+ \\
+\left(E_{n_{0}, \Delta_{z}^{\prime}}^{s, \xi}-E_{n_{0}, \Delta_{z}}^{s, \xi}\right)\left(n-n_{0}\right)+ \\
+\frac{1}{2}\left(E_{n_{0}, \Delta_{z}^{\prime}, \Delta^{\prime}}^{s, j}-E_{n_{0}, \Delta_{z}}^{s, \xi^{\prime \prime}}\right)\left(n-n_{0}\right)^{2}
\end{array}
$$

where primes in energies denote derivatives with respect to level numbers.

Therefore, the exponential in (17) contains three parts. The first one does not depend on $n$ and leads to a global phase factor, while the other two ones give rise to periodic oscillations with two different periods: The first oscillation period is analogous to the one found when a localized wave packet is excited with an energy spectrum which is tightly spread around a large central value of the principal quantum number of the unperturbed Hamiltonian. Following correspondence principle arguments it has been associated with the classical period of motion of the unperturbed Hamiltonian [39]. Therefore, in our case it can be called classical Loschmidt period,

$$
T_{\mathrm{L}}^{\mathrm{Cl}}=\frac{2 \pi \hbar}{\left|E_{n_{0}, \Delta_{z}^{\prime}}^{s, \xi}-E_{n_{0}, \Delta_{z}}^{s, \xi}\right|} .
$$

The second oscillation period is responsible for the long-term spreading and reconstruction of the wave packet associated with the quantum revival time scale [39] and can be called revival Loschmidt period

$$
T_{\mathrm{L}}^{\mathrm{R}}=\frac{2 \pi \hbar}{\mid E_{n_{0}, \Delta_{z}^{\prime}}^{s, \xi \prime \prime}-E_{n_{0}, \Delta_{z}}^{s, \xi \prime \mid}} .
$$

An analogous derivation can be made for the time evolution of the fidelity for a Type II wave packet, but in this case a new feature arises. Since we have both positive and negative values for $n$, the absolute values in the Kronecker deltas in Eq. (18) become relevant and the time evolution is now governed by the energy differences $E_{n, \Delta_{z}^{\prime}}^{s, \xi}-E_{m, \Delta_{z}}^{s, \xi}$ for $m= \pm n$. 
This means the exponential in the equation analogous to (17) no longer contains a global phase factor but a third period of oscillation -similar to the one due to Zitterwebegung that appears for the unperturbed Hamiltonian [40]- that we can call Zitterbewegung Loschmidt period,

$$
T_{\mathrm{L}}^{\mathrm{Zb}}=\frac{\pi \hbar}{\left|E_{n_{0}, \Delta_{z}^{\prime}}^{s, \xi}-E_{n_{0}, \Delta_{z}}^{s, \xi}\right|} .
$$

The overall situation for this Loschmidt periodicities is analogous to the case of quantum revivals in monolayer graphene quantum dots as well as monolayer and bilayer graphene rings $[41,42]$. Classical and revival periodicities appear for both Type I and Type II packets while Zitterbewegung only appears for Type II packets.

For the sake of simplicity we are going to select one valley $(\xi=1)$ and one spin orientation $(s=1)$ to study these oscillations in the next sections. Analogous results can be obtained for other $\{s, \xi\}$ combinations.

\section{LOSCHMIDT PERIODICITIES}

As we pointed out in the Introduction, it has been proposed that silicene undergoes a topological phase transition at the charge neutrality point $\Delta_{z}^{(0)}=s \xi \Delta_{\text {so }}$. One could expect the phase transition at this point to show as divergences in the three periodic oscillations considered. We present in Fig. 3 Zitterbewegung, classical and revival Loschmidt periods for three different $\delta_{z}$ values for a Type II wave packet (the last two are also valid for a Type I packet).

It is clear that the the three periodicities diverge near the charge neutrality point but not exactly at it. In fact there seems to be a dependence on the value of $\delta_{z}$ : The smaller $\delta_{z}$ the closer the divergence is to the CNP. There is an easy explanation for this feature. We have to take into account that in order to calculate the fidelity we are working with two slightly different energy spectra corresponding to two different electric field values $\Delta_{z}$ and $\Delta_{z}^{\prime}=\Delta_{z}+\delta_{z}$. For instance, for Eq. (22) to diverge, we need $E_{n, \Delta_{z}^{\prime}}^{s, \xi}=E_{m, \Delta_{z}}^{s, \xi}$ and using (2), this means $\left(\Delta_{s, \xi}^{\prime}\right)^{2}=\Delta_{s, \xi}^{2}$, that leads to $\left|\Delta_{z}^{\prime}-s \xi \Delta_{\mathrm{so}}\right|=\left|\Delta_{z}-s \xi \Delta_{\mathrm{so}}\right|$ or, equivalently, $\left|\Delta_{z}+\delta_{z}-\Delta_{z}^{(0)}\right|=\left|\Delta_{z}-\Delta_{z}^{(0)}\right|$. Disregarding the trivial solution $\delta_{z}=0$, we get $\Delta_{z}+\delta_{z}-\Delta_{z}^{(0)}=$ $-\left(\Delta_{z}-\Delta_{z}^{(0)}\right)$ and, finally, the critical point is located at

$$
\frac{\Delta_{z}}{\Delta_{z}^{(0)}}=1-\frac{1}{2} \frac{\delta_{z}}{\Delta_{z}^{(0)}} .
$$



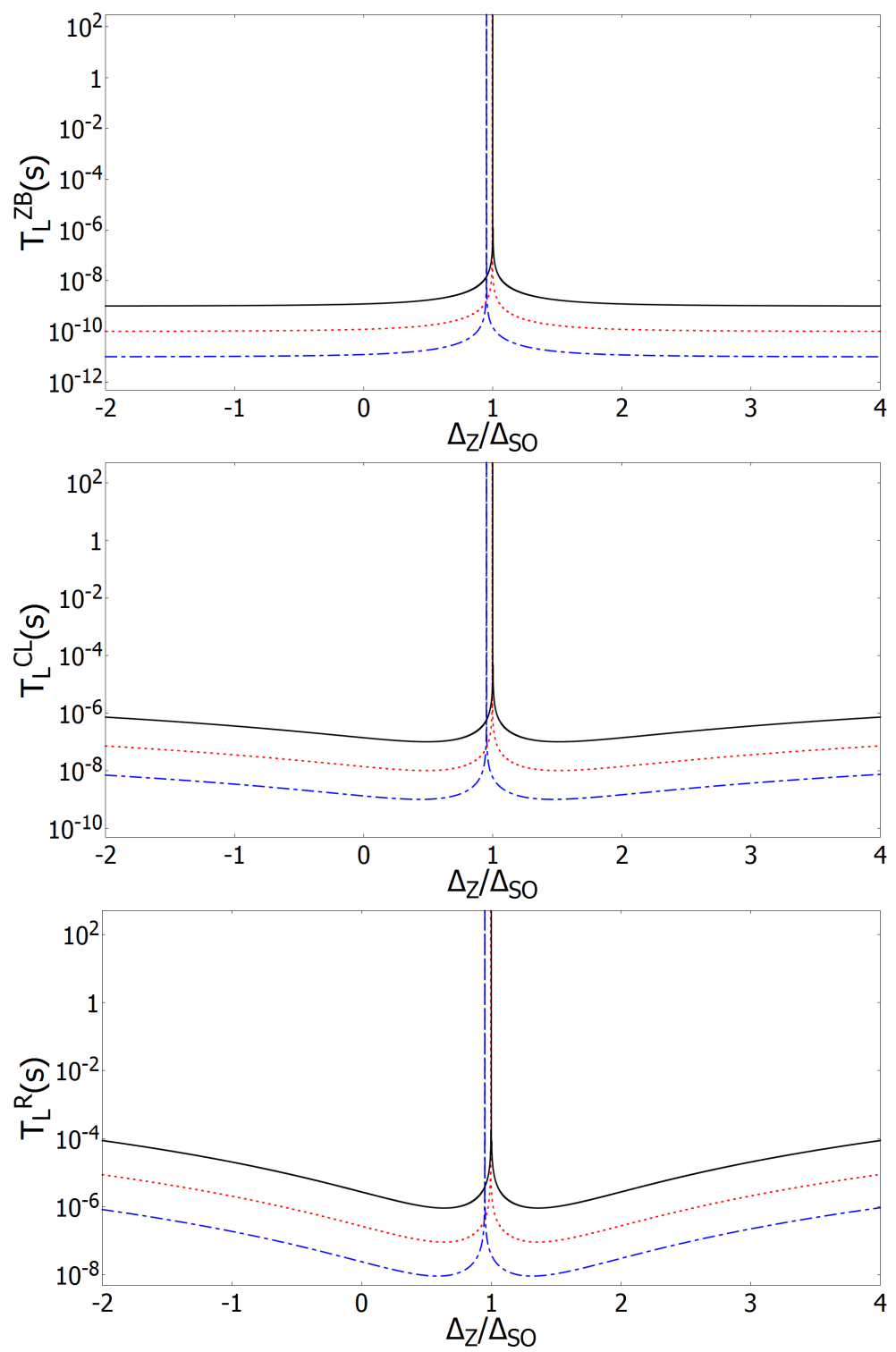

FIG. 3: Zitterbewegung (upper panel), classical (intermediate panel) and revival (lower panel) Loschmidt periods for different $\delta_{z}$ values for a Type II wave packet (the last two are also valid for a Type I packet). Solid black line for $\delta_{z}=10^{-3} \Delta_{\text {so }}$, red dotted line for $\delta_{z}=10^{-2} \Delta_{\text {so }}$ and blue dash-dotted line for $\delta_{z}=10^{-1} \Delta_{\mathrm{so}}$.

This result indicates that the divergences in the Lochsmidt periods should appear close to the CNP (because $\delta_{z} \ll \Delta_{z}^{(0)}$ ) but not exactly at that point. This displacement with respect to the exact critical point appears because in order to calculate Loschmidt fidelity we are using at the same time the unperturbed and the perturbed Hamiltonians corresponding to two slightly different electric fields and, therefore, slightly different charge neutrality points. 
The smaller the difference in the electric fields, the closer the divergence is to the CNP of the unperturbed Hamiltonian.

Taking into account the valley and spin selected $(\xi=s=1), \Delta_{z}^{(0)}=s \xi \Delta_{\text {so }}=\Delta_{\text {so }}$ and Eq. (23) reads

$$
\frac{\Delta_{z}}{\Delta_{\mathrm{so}}}=1-\frac{1}{2} \frac{\delta_{z}}{\Delta_{\mathrm{so}}}
$$

We present in Fig. 4 Zitterbewegung, classical and revival Loschmidt periods for the same three different $\delta_{z}$ values but only in the vicinity of the CNP. The three panels in the figure look similar but the time scales are different, $T_{\mathrm{L}}^{\mathrm{Zb}} \ll T_{\mathrm{L}}^{\mathrm{Cl}} \ll T_{\mathrm{L}}^{\mathrm{R}}$. It is clear that Eq. (24) is satisfied.

All Loschmidt periodicities shown in Fig. 4 seem to be symmetric with respect to the divergence. We will test in the next section if that is the case and analyze in greater detail the time evolution of the wave packets.

\section{TIME-EVOLUTION OF THE ECHOES}

We present in this section the time-dependent fidelity $M_{\mathrm{L}}(t)$ defined in Eq. (16) and the time-independent fidelity calculated using Eq. (8), $F=M_{\mathrm{L}}(t=0)$, when $\delta_{z}=10^{-2} \Delta_{\text {so }}$. Similar results are found for the $\delta_{z}=10^{-1} \Delta_{\text {so }}$ and $\delta_{z}=10^{-3} \Delta_{\text {so }}$ cases.

\section{A. Type I wave packets}

Let's analyze first the region below the critical point that, with the parameters chosen, is located at $\Delta_{z}=0.995 \Delta_{\text {so }}$. Fig. 5 shows the time evolution of the fidelity for the Type I packet described in the upper panel of Fig. 2 for three different values of the electric field $\left(\Delta_{z}=-2 \Delta_{\mathrm{so}}, \Delta_{z}=0\right.$ and $\left.\Delta_{z}=0.9 \Delta_{\mathrm{so}}\right)$. In each case, the fidelity is shown as a function of time and as a function of classical and revival Loschmidt periods.

If we compare the upper left graph in each panel we can see that the classical Loschmidt period decreases when $\Delta_{z}$ increases from $-2 \Delta_{\text {so }}$ to 0 but increases when it goes from 0 to $0.9 \Delta_{\text {so }}$. In fact, if we look at the central panel in Fig. 3 we can see that $T_{\mathrm{L}}^{\mathrm{Cl}}$ decreases down to a minimum and then starts to increase when the system approaches the critical point.

If we now focus our attention on the upper right graph in each panel where $M_{\mathrm{L}}(t)$ is depicted as a function of time but in $T_{\mathrm{L}}^{\mathrm{Cl}}$ units we notice that, obviously, there is one 

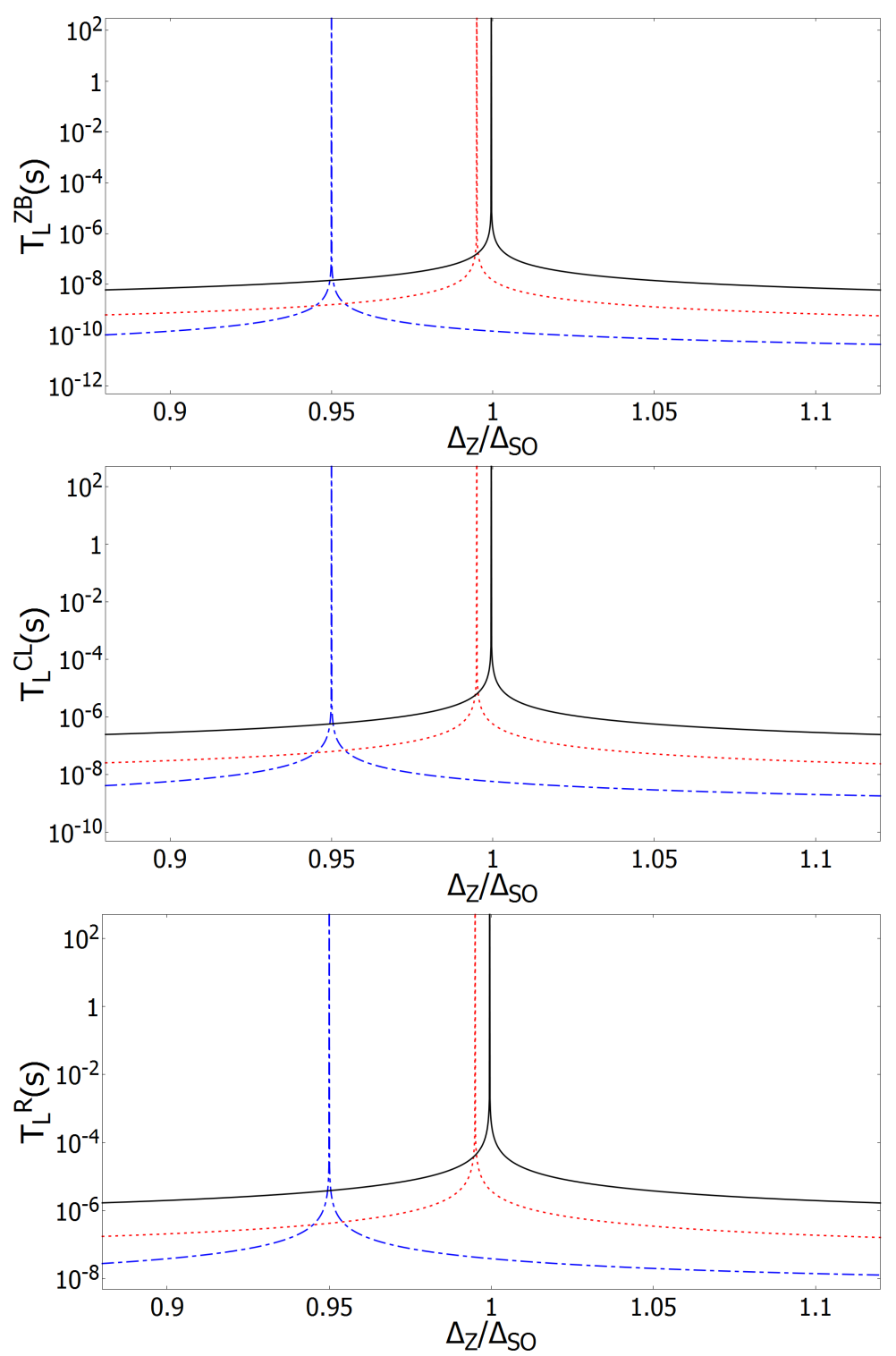

FIG. 4: The same as Fig. 3 but in the vicinity of the charge neutrality point.

oscillation per unit in the $t$-axis but that while the amplitude of the oscilllations seems to be constant in the upper panel, it is clearly modulated in the other two cases: It oscillates one and a half times in ten classical periods for $\Delta_{z}=0.9 \Delta_{\mathrm{so}}$ but only describes half an oscillation when $\Delta_{z}=0$. Nevertheless, if we use Loschmidt revival period as time unit we get the graphs in the lower part of each panel. It is clear that there is always amplitude modulation but that when $\Delta_{z}=-2 \Delta_{\text {so }}$ a great number of classical oscillations occur before a revival oscillation completes. In fact, the lower panel in Fig. 3 looks similar to the middle one but there is a big difference: the slope of the decreasing part is much bigger in absolute 

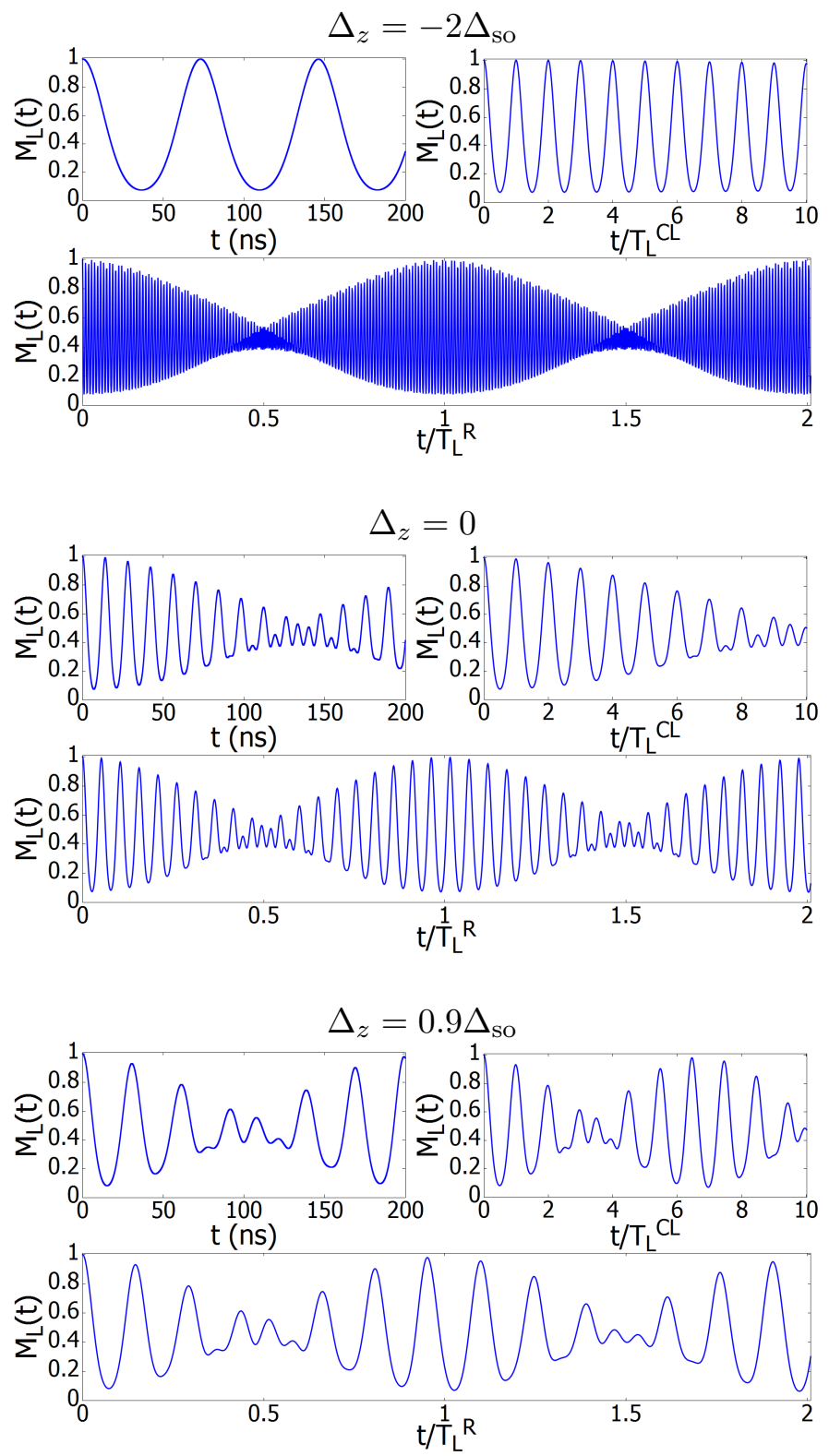

FIG. 5: Time-dependent fidelity for a Type I packet for three different values of $\Delta_{z}$ : $-2 \Delta_{\text {so }}$ in the upper panel, 0 in the middle panel and $0.9 \Delta_{\text {so }}$ in the lower one. In each case, this quantity is shown as a function of time and as a function of classical and revival Loschmidt periods.

value (remember the logarithmic scale).

Let's move now to the region above the critical point. To test if classical and revival Loschmidt periods are symmetric with respect to this point as Fig. 3 suggests we have calculated the $\Delta_{z}=3.99 \Delta_{\mathrm{so}}, \Delta_{z}=1.99 \Delta_{\text {so }}$ and $\Delta_{z}=1.09 \Delta_{\text {so }}$ cases that are the symmetrical ones to those presented in Fig. 5. The results are exactly the same as those of their coun- 
terparts and we don't show them. In fact, we have studied 9 different pairs of symmetrical points, both close to and far from the critical point, and in all cases there is perfect agreement. It is then clear than both classical and revival Loschmidt periods are symmetric with respect to the critical point as the middle and lower panels in Fig. 3 suggest.

In order to analyze in greater detail the amplitude of the oscillations in the fidelity we have calculated the maximun amplitude of the time-dependent fidelity,

$$
\left(A_{M_{\mathrm{L}}}\right)_{\mathrm{MAX}}=\max _{t \in\left[0, T_{\mathrm{L}}^{\mathrm{R}}\right)} M_{\mathrm{L}}(t) .
$$

The left panel in Fig. 6 represents this quantity as a function of the electric field. The maximum amplitude slightly decreases close to the critical point and it is symetric with respect to it.

We have also calculated the time-independent fidelity, $F$ and plotted it in the right panel in Fig. 6. The result is the same, indicating that the maximum in fidelity occurs at $t=0$.
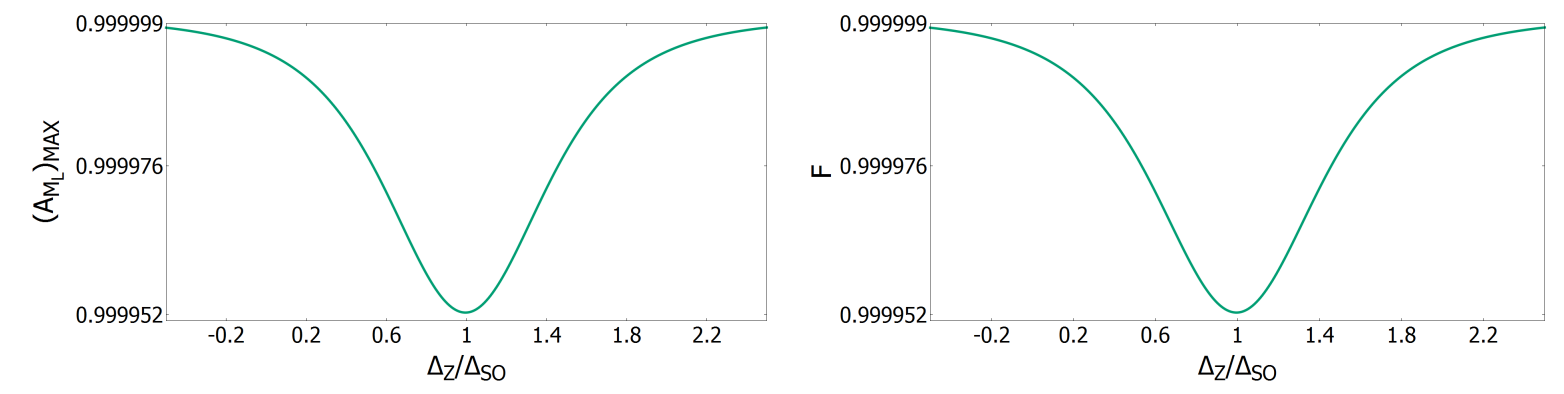

FIG. 6: Maximun amplitude of the time-dependent fidelity (left panel) and time-independent fidelity (right panel) for a Type I packet with $\delta_{z}=10^{-2} \Delta_{\text {so }}$.

\section{B. Type II wave packets}

Cat states give rise to a third periodicity due to Zitterbewegung with a shorter period than the classical one and this fact introduces some differences with respect to Type I packets.

Let's start again with the region below the critical point. Fig. 7 shows the time evolution of the fidelity for the Type II packet described in the lower panel of Fig. 2 for three different values of the electric field $\left(\Delta_{z}=-2 \Delta_{\text {so }}, \Delta_{z}=0\right.$ and $\left.\Delta_{z}=0.9 \Delta_{\text {so }}\right)$. In each case, the fidelity is shown as a function of time and as a function of Zitterbewegung, classical and revival Loschmidt periods. 

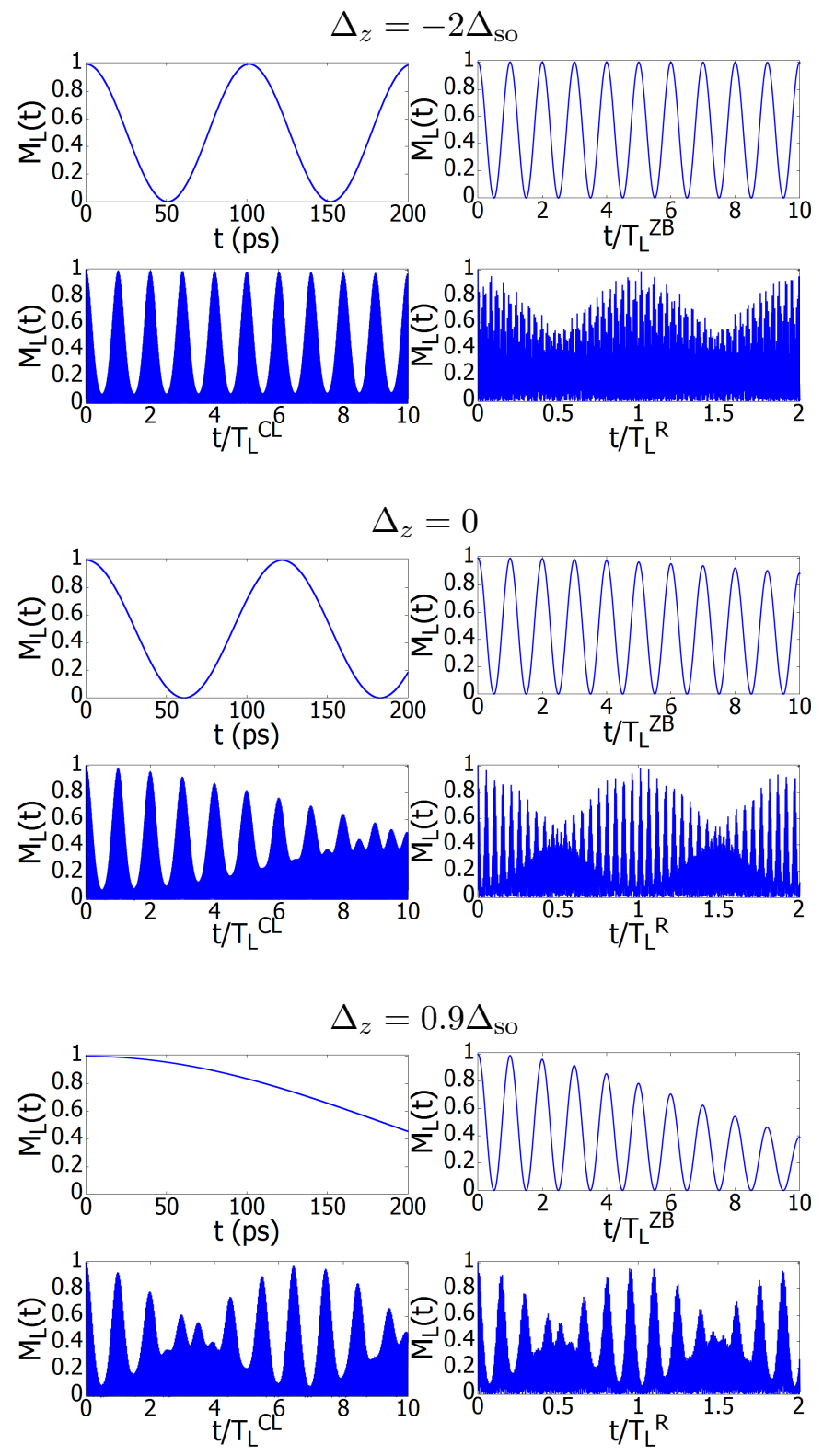

FIG. 7: Time-dependent fidelity for a Type II packet for three different values of $\Delta_{z}$ : $-2 \Delta_{\text {so }}$ in the top panel, 0 in the central panel and $0.9 \Delta_{\text {so }}$ in the bottom one. In each case, this quantity is shown as a function of time and as a function of Zitterbewegung, classical and revival Loschmidt periods.

If we compare the upper left graph in each panel we can see that the Zitterbewegung Loschmidt period increases as $\Delta_{z}$ increases from $-2 \Delta_{\text {so }}$ to $0.9 \Delta_{\text {so }}$. If we look at the upper panel in Fig. 3 we can see that $T_{\mathrm{L}}^{\mathrm{Zb}}$ increases monotonically up to the critical point.

If we now move to the upper right graph in each panel where $M_{\mathrm{L}}(t)$ is depicted as a 
function of time but in $T_{\mathrm{L}}^{\mathrm{Zb}}$ units we see something similar to what happened when Type I results were plotted using $T_{\mathrm{L}}^{\mathrm{Cl}}$ as time unit but the decay in the amplitude is much slower. More oscillations are needed for the amplitude modulation to show.

If we use Loschmidt revival period as time unit we get the graphs in the lower left part of each panel. The modulation is now more clear but Zitterbewegung is so fast with this time scale that indivual oscillations are no longer visible and become a solid area. This is also the case when $T_{\mathrm{L}}^{\mathrm{R}}$ is used as time unit (lower right graph of each panel). Nevertheless, Zitterbewegung, in spite of introducing a new oscillation that exhibits a different behavior with electric field than classical or revival oscillations (compare the upper panel in Fig. 3 with the other two ones), doesn't perturbate classical and revival Loschmidt periods because it has a completely different time scale.

Focusing now on the region above the critical point, we have performed the same test as we did for Type I packet and the result holds. Not only classical and revival Loschmidt periods are symmetric with respect to the critical point but Zitterbewegung Loschmidt period also exhibits the same symmetry.

We have also calculated the maximun amplitude of the time-dependent fidelity defined in (25). The left panel in Fig. 8 represents this quantity as a function of the electric field. The maximum amplitude slightly decreases in the vicinity of the critical point but increases close to it and presents the absolute maximum at this critical point. This is different to what happened for Type I packets but the symmetry with respect to the critical point is preserved.

We have calculated the time-independent fidelity defined in (8) too and plotted it in the right panel in Fig. 8. The result doesn't coincide with that for $\left(A_{M_{\mathrm{L}}}\right)_{\mathrm{MAX}}$ but with the one for $F$ in Type I packets. This difference indicates that the maximum in fidelity doesn't appear at $t=0$ for cat states. Zitterbewegung can't affect $F$ because this quantity only has to do with the initial overlap of the wave functions corresponding to the slighly different electric fields. But it changes $\left(A_{M_{\mathrm{L}}}\right)_{\mathrm{MAX}}$ as the left panels in Figs. 6 and 8 clearly show.

\section{CONCLUSIONS}

We have studied Zitterbewegung, classical and revival periods for silicene as a function

of electric field intensity when the electric field is changed by a very small amount. We have 

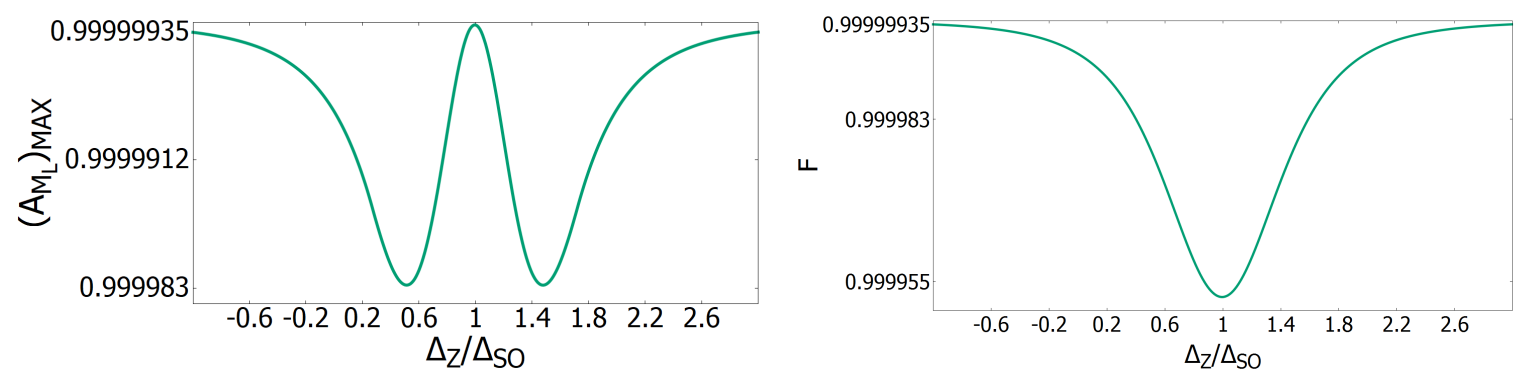

FIG. 8: Maximun amplitude of the time-dependent fidelity (left panel) and time-independent fidelity (right panel) for a Type II packet with $\delta_{z}=10^{-2} \Delta_{\text {so }}$.

used two different kinds of wave packets. Type I packet is a simple Gaussian while Type II is a double Gaussian cat state. We have shown that all three Loschmidt periods diverge close to the charge neutrality point. The offset with respect to this point is determined by the amount the electric field is changed.

We have also proven that time-independent as well as time-dependent fidelities can be used as markers of topological phase transitions in silicene (and other 2D gapped materials) and that time-indepent fidelity is equal to the maximum of the time-dependent one for Type I packets but not for Type II packets.

\section{Acknowledgments}

This research was supported by the Junta de Andalucía projects FQM.1861 and FQM381, the EU-funded Hungarian grant EFOP-3.6.2-16-2017-00005 and the National Research, Development and Innovation Fund of Hungary, financed under 123988 funding scheme.

\section{Bibliography}

[1] K. Takeda and K. Shiraishi, Phys. Rev. B 50, 14916 (1994).

[2] G. G. Guzman-Verri and L. Lew Yan, Phys. Rev. B 76, 075131 (2007).

[3] P. Vogt et al., Phys. Rev. Lett. 108, 155501 (2012). 
[4] B. Augray et al., App. Phys. Lett. 96, 183102 (2010).

[5] B. Lalmi et al., App. Phys. Letters 97, 223109 (2010).

[6] A. Feurence et al., Phys. Rev. Lett. 108, 245501 (2012).

[7] P. E. Padova et al., App. Phys. Lett. 96, 261905 (2010).

[8] W-F. Tsai et al., Nat. Commun. 4, 1500 (2013).

[9] M. Tahir and U. Schwingenschlögl, Scientific Reports, 3, 1075 (2013).

[10] E. Romera and M. Calixto, EPL, 111, 37006 (2015).

[11] M. Calixto and E. Romera, Journal of Statistical Mechanics, P06029 (2015).

[12] E. Romera and M. Calixto, Journal of Physics: Condensed Matter,27, 175003 (2015).

[13] M. Calixto and E. Romera, EPL, 109, 40003 (2015).

[14] E. Romera et al., EPL, 115, 20008 (2016).

[15] A. Peres, Phys. Rev. A, 34, 1610 (1984).

[16] P. Jacquod, I. Adagideli and C. W. J. Beenakker, Phys. Rev. Lett. 89, 154103 (2002).

[17] T. Prosen, T. H. Seligman and M. Znidaric, Prog. Theor. Phys. Suppl. 150, 200 (2003).

[18] M. Gutiérrez and A. Goussev, Phys. Rev. E 79, 046211 (2009).

[19] F. M. Cucchietti, H. M. Pastawski and R. A. Jalabert, Phys. Rev. B 70, 035311 (2004).

[20] E. J. Torres-Herrera and L. F. Santos, Phys. Rev. E 89, 062110 (2004).

[21] W. W. Ho and D. A. Abanin, Phys. Rev. B 95, 094302 (2017).

[22] T. Gorin et al., Phys. Rep. 435, 33 (2006).

[23] M. A. Nielsen and I. L. Chuang, Quantum computation and quantum information (Cambridge University Press, Cambridge) 2000.

[24] M. Serbyn and Dmitry A. Abanin, Phys. Rev. B 96, 014202 (2017).

[25] R. Jafari and H. Johannesson, Phys. Rev. Lett. 118, 015701 (2017).

[26] H. T. Quan, Z. Song, X. F. Liu, P. Zanardi, and C. P. Sun, Phys. Rev. Lett. 96, 140604 (2006).

[27] Zi-Gang Yuan, Ping Zhang, and Shu-Shen Li, Phys. Rev. A 75, 012102 (2007).

[28] J. Häppölä, G. Halász, and A. Hamma, Phys. Rev. A 85, 032114 (2012).

[29] R. Jafari and Henrik Johannesson, Phys. Rev. B 96, 224302 (2017).

[30] S. Trivedi, A. Srivastava and R. Kurchania, J. Comput. Theor. Nanosci. 11, 1-8 (2014).

[31] B. van den Broek et al. 2D Materials 1, 021004 (2014).

[32] N. D. Drummond, V. Zólyomi, and V. I. Fal'ko, Phys. Rev. B 85, 075423 (2012). 
[33] C. C. Liu, W. Feng and Y. Yao, Phys. Rev. Lett. 107, 076802 (2011).

[34] C. C. Liu, H. Jiang and Y. Yao, Phys. Rev. B 84, 195430 (2011).

[35] L. Stille, C. J. Tabert and E. J. Nicol, Phys. Rev. B 86, 195405 (2012); C.J. Tabert and E.J. Nicol, Phys. Rev. Lett. 110, 197402 (2013); C.J. Tabert and E.J. Nicol, Phys. Rev. B 88, 085434 (2013).

[36] T. Gorina et al., Physics Reports 435, 33 (2006).

[37] Gu Shi-Jian, Chin. Phys. Lett., 26, 026401 (2009).

[38] M. Ezawa, New Journal of Physics 14, 033003 (2012).

[39] R.W. Robinett, Phys. Rep., 392, 1 (2004).

[40] E. Romera and F. de los Santos, Phys. Rev. B, 80, 165416 (2009).

[41] T. García et al., J. Phys.: Condens. Matter, 25, 235301 (2013).

[42] T. García, N. A. Cordero and E. Romera, Phys. Rev. B, 89, 075416 (2014). 